\title{
La imagen de la mujer en las crónicas de Carmen de Burgos Colombine durante la Guerra de Marruecos
}

\author{
Adriana PAíno Ambrosio \\ Universidad de Salamanca \\ adriana.paino@usal.es \\ Lucía JiMÉNEZ IGLESIAS \\ Universitat de Barcelona \\ luciajimenez@ub.edu \\ $\mathrm{M}^{\mathrm{a}}$ Isabel RoDRÍGUEz FIDALGO \\ Universidad de Salamanca \\ mrfidalgo@usal.es
}

Recibido: 21 de junio de 2016

Aceptado: 12 de septiembre de 2016

\begin{abstract}
Resumen
Traductora, novelista, crítica de arte, defensora de los derechos de la mujer y del divorcio, maestra... son muchas las facetas que caracterizan a Carmen de Burgos, pero si hay una que destaca por encima de las demás es la de periodista. Carmen ha pasado a la historia por ser la primera mujer contratada como redactora en un periódico de España. Frecuentemente conocida bajo el sobrenombre de "Colombine", esta comunicadora del siglo XX destacó por su ideología liberal que transgredía las convenciones morales de la época. Este trabajo realiza una revisión de la producción periodística de Carmen de Burgos en su etapa como corresponsal de guerra en Marruecos y analiza el tratamiento que la autora realiza sobre la mujer en sus crónicas durante los meses de agosto y septiembre de 1909.
\end{abstract}

Palabras clave: Carmen de Burgos; Colombine; periodismo; Diario Universal; Heraldo de Madrid; Guerra de Marruecos.

\section{Women's representation in Carmen de Burgos Colombine's reports during Marruecos War}

\begin{abstract}
Translator, writer, film critic, women's rights and divorce advocate, teacher... There are many facets that portray Carmen de Burgos but one of them stands out among the others: she was a journalist. Carmen has gone down in history because she was the first woman being hired as a writer by a newspaper in Spain. Known by her pen-name Colombine, this 20th century communication professional stood out due to her liberal thinking which disagreed with the moral standards of her age. This paper looks over Carmen de Burgos' journalistic production as Marruecos war correspondent in order to analyse how the author presents women in her articles during August and September, 1909.
\end{abstract}

Keywords: Carmen de Burgos; Colombine; journalism; Diario Universal; Heraldo de Madrid; Marruecos War. 


\section{Referencia normalizada}

Paino Ambrosio, A., Jiménez Iglesias, L. y Rodríguez Fidalgo, Ma ${ }^{\mathrm{a}}$ I. (2016). La imagen de la mujer en las crónicas de Carmen de Burgos Colombine durante la Guerra de Marruecos. Historia y Comunicación Social. Vol 21, número 2, páginas 413-432.

Sumario: 1. Introducción; 1.1. Las mujeres en el periodismo español entre finales del siglo XIX y principios del XX;1.2. La representación de la mujer en la prensa a comienzos del siglo XX; 2. Carmen de Burgos, Colombine y el periodismo; 2.1. La relación de Carmen con el periodismo; 3. Metodología; 3.1. Hipótesis y objetivos de investigación; 3.2. Planteamiento del análisis; 4. Análisis y resultados; 4.1. Análisis del tratamiento periodístico; 4.2. Tratamiento de la mujer en las crónicas de Colombine; 5. Conclusiones; Referencias bibliográficas.

\section{Introducción}

1.1. Las mujeres en el periodismo español entre finales del siglo XIX y principios del XX

La profesión periodística hasta bien entrado el siglo XIX estaba mayoritariamente reservada, como muchos otros oficios, al sexo masculino, y apenas había lugar para las mujeres que querían participar en la vida cultural y política. Si bien es verdad que la mentalidad de esta época comenzaba a aceptar el papel cada vez más activo de la mujer en la sociedad, no es menos cierto que la pervivencia de ciertas estructuras propias del Antiguo Régimen impedían su desarrollo pleno en el ámbito profesional. La educación también estaba diferenciada en función del género, y la vida de las mujeres en ese momento estaba ligada al hogar y el cuidado de los hijos. No obstante, a pesar de la restricción existente y debido a la creciente aceptación del rol laboral de la mujer propia de esta época, se pueden encontrar huellas de la participación femenina en la prensa desde el siglo XVII con nombres como el de Francisca de Aculodi, quien reproducía cada quince días Noticias principales y verdaderas, una traducción de una gaceta publicada en Bruselas (Bernárdez, de Andrés, Lorenzo, y Vargas, 2007); y Beatriz Cienfuegos que, ya en el siglo XVIII, publicaba el semanario $L a$ pensadora gaditana, aunque en este caso su autoría está cuestionada y hay quien se la atribuye al clérigo andaluz Juan Francisco de Postigo (Dale, 2005). Como ellas, hubo otras mujeres precursoras de la incorporación de la mujer al oficio periodístico y que abrieron brecha para la normalización de la presencia femenina en los medios de comunicación, aunque cuando realmente se va a producir un cambio significativo en este sentido es entre el último tercio del siglo XIX y el primero del siglo XX, y es que como señalan Bernárdez et al. "el desarrollo de los derechos para las mujeres, su acceso a la cultura, al voto o a la educación normalizada corren paralelos con la expansión del periodismo en España" (2007: 22).

Si bien Carmen de Burgos (1867-1932) no es la primera mujer que ejerció el periodismo en España, sí que es la primera que obtuvo un puesto de redactora en un periódico español, algo que ocurre, como se verá en el siguiente epígrafe, justo después 
del cambio de siglo, cuando se incorpora a la redacción de Diario Universal. Emilia Pardo Bazán, Carmen Eva Nelken o Josefina Carabias, entre muchas otras, vienen a sumarse a la lista de pioneras que dedicaron su vida, o al menos una parte de ella, a la comunicación en las primeras décadas del siglo XX.

\subsection{La representación de la mujer en la prensa a comienzos del siglo $\mathrm{XX}$}

La prensa ha ejercido tradicionalmente y ejerce hoy en día un papel fundamental en la conformación de la opinión pública. Si bien las circunstancias históricas y sociales del periodo en el que Carmen de Burgos desarrolló su labor periodística no facilitaban la cantidad de información y el acceso a los contenidos informativos propios de la actualidad, no se debe menospreciar la influencia de los periódicos en la construcción de la imagen pública de la mujer.

En su análisis comparado de los períodos 1910-1915 y 2000-2005, Rocío Herrero (2010) establece los principales rasgos de la representación de la mujer en la prensa de ambas épocas. La autora destaca cómo durante los dos periodos la mujer aparece subordinada al hombre y asociada a trabajos que tienen que ver con tareas del hogar, así como con profesiones tradicionalmente asociadas con el género femenino: mecanógrafa, taquígrafa o bordadora. Herrero afirma que “(...) no se concebía para ella más educación que la que corresponde a su papel de madre y que garantiza el mantenimiento de las relaciones de género tradicionales" (2010: 607), y que socialmente se relacionaba a la mujer con actividades como ir de compras, pasear o formar parte de actos benéficos.

En esta misma línea, Nuria Rodríguez (2007) destaca la utilización de la imagen de la mujer como reclamo en los carteles publicitarios del primer tercio del siglo XX. Hasta 1910 aproximadamente, la autora señala que la publicidad en prensa era muy escasa en España (a partir de esa fecha comenzó a aumentar paulatinamente el número de anuncios en los periódicos), siendo los carteles los principales transmisores de los mensajes publicitarios a través de la imagen de la mujer como ser bello y exótico.

\section{Carmen de Burgos, Colombine y el periodismo}

“Como mujer vivió muchos de los males machistas de la época, como periodista denunció todo tipo de injusticia social en miles de artículos, como novelista escribió más de 150 obras comprometidas con los ideales de igualdad, como política luchó abiertamente en busca de la injusticia social y como pedagoga creía firmemente en que la educación de la mujer era la solución para muchos de los males sociales" (Latorre, 1998: 173).

Carmen de Burgos Seguí fue una pionera en muchos aspectos, tanto de su vida personal como de su trabajo. Firme defensora de los derechos de la mujer y de la ley del divorcio, representó un ejemplo de modernidad y de progreso en su época 
(Núñez, 2010). Sin embargo, como muchas otras mujeres de su generación, ha sido una de las grandes olvidadas a la hora de citar a los personajes que forman parte de la cultura española de comienzos del siglo XX (Latorre, 1998); un olvido que se debe en parte a sus ideas progresistas que chocaron de frente con el control ideológico del régimen franquista que silenció durante años su labor como escritora y periodista (Sevillano \& Segura, 2005; Cabanillas, 2006; Latorre, 1998). Esto no ha impedido sin embargo el posterior reconocimiento de su trabajo; buena prueba de ello es que en las dos últimas décadas Carmen de Burgos ha sido objeto de una gran atención para diferentes autores, lo que ha permitido rescatar su trayectoria del olvido y poner en valor la importancia que ha tenido esta figura desde el punto de vista literario y periodístico (Sevillano \& Segura, 2005).

Carmen de Burgos nació en 1867 en una familia acomodada y pasó su infancia en el valle de Rodalquilar (Almería). Se casó a los dieciséis años con Arturo Álvarez Bustos, un periodista doce años mayor que ella (Núñez, 1992). Un matrimonio que al poco tiempo fracasó y que como sostiene Bárbara Minesso "se convierte pronto para ella en una pesadilla: a los vicios, infidelidades y depravación del marido se une la tragedia de ver morir uno después de otro a varios hijos" (2011: 174). Algunos años más tarde, en 1903, cuando ya era redactora en el Diario Universal, Carmen decidió realizar una encuesta para que los lectores mostraran su apoyo u oposición a la aprobación de una supuesta ley del divorcio (Scanlon, 1986). La idea surgió a raíz de la aparición de un "Club de matrimonios mal avenidos" en Madrid. Dado el interés suscitado entre el público por su encuesta (intelectuales como Pío Baroja, Vicente Blasco Ibáñez o Giner de los Ríos enviaron misivas expresando su opinión), Carmen de Burgos reunió todas las cartas recibidas y las publicó en El Divorcio en España (1904). A pesar de que la mayoría de los participantes se mostraron favorables al divorcio, lo cierto es que se aún quedaban algunos años para su normalización. La Real Orden de 27 de agosto de 1906 facilitaba ligeramente el matrimonio civil, pero causó una ardua campaña de críticas y fue derogada a principios de 1907 (Scanlon, 1986). Se trataba, por tanto, de una opción casi impensable para la gran mayoría de las mujeres de la época.

Cuando Carmen de Burgos decide liberarse de su desafortunado matrimonio lo hace a través de la formación académica. Decidió estudiar para ser maestra, obteniendo en 1895 la titulación de Maestra Primera de Enseñanza Elemental y tres años más tarde la de Enseñanza Superior (Núñez, 1992). Sus estudios la permitirán lograr la independencia económica y abandonar su ciudad natal (en compañía de su hija María), pero, además, como señala Concepción Núñez, le servirán para adquirir no solo "una concepción del mundo sino una actitud frente a él" (en Burgos, 1989: 14). 


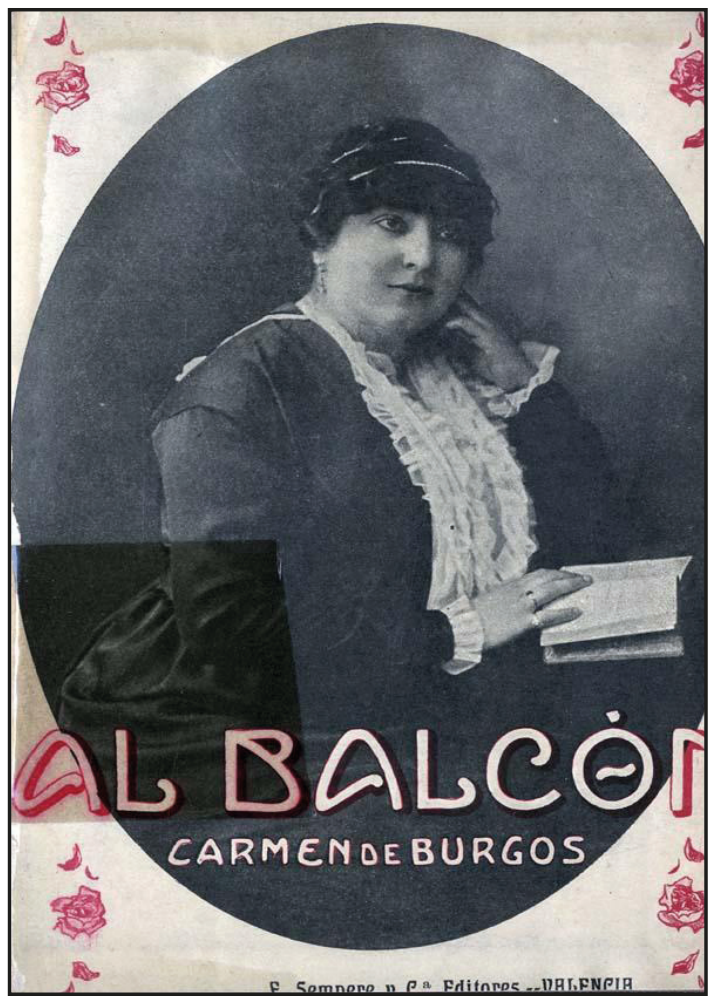

Fig. 1. Portada de Al balcón (1913) donde aparece un retrato de Carmen de Burgos. Fuente: http://goo.gl/820eh6

Carmen ejerció como profesora en la Escuela Normal de Maestras de Guadalajara hasta 1901, año en que se traslada a Madrid y comienza a ser conocida por su actividad periodística y literaria. Si bien hay muchos aspectos destacables de la biografía de Carmen de Burgos antes de su llegada a la capital española este viaje marcará un momento trascendental en su vida y, especialmente, en su trayectoria profesional por la que hoy día es recordada y reconocida.

\subsection{La relación de Carmen con el periodismo}

"Empecé por cajista de imprenta, en la que poseía mi padre político. Después escribí con las tijeras para completar un periódico satírico. Mi primer artículo mereció los honores de la crítica y la reproducción fuera de la provincia... Luego, circunstancias tristes de la vida, que pertenecen al sagrado de la familia y no quiero recordar, me impulsaron por la senda del Arte y del Trabajo" (Burgos de, 1913: 11).

Sus primeros contactos con la profesión tras su llegada a Madrid comienzan en 1902, cuando aparecen algunos artículos publicados en La Correspondencia de España y en El Globo (Sevillano \& Segura, 2009; Núñez, 1992). Este mismo año comienza su columna Notas Femeninas, en relación a la situación de la mujer espa- 
ñola. Pero cuando realmente se consagra como periodista es con su incorporación a la redacción del Diario Universal en 1903, que la convirtió en la primera mujer que alcanzaba este puesto en un periódico en España (Establier, 1997). En este medio, que dirigía Augusto Suárez Figueroa, escribía diariamente una columna titulada Lecturas para la mujer, sobre temas relacionados con la mujer, la moda o la maternidad (Suárez, 2010). "Inicia así una trayectoria como periodista profesional que se prolongará hasta el final de su vida, y que irá incrementando a medida que pasen los años" (Sevillano \& Segura, 2009: 21).

En Diario Universal nace el seudónimo que utilizará Carmen y por el que será conocida el resto de su vida: "Colombine" (Núñez, 2012), un nombre que hace referencia a un personaje de la Comedia del arte (Sanz, 2010; Latorre, 1998) aunque no será el único que utilice ya que también llega a firmar con otros sobrenombres como "Perico de los Palotes", Gabriel Luisa o Marianella, entre otros (Latorre, 1998). También en 1902 inicia su colaboración en $A B C$ con una columna fija que se prolongará hasta 1905, en ella, según apunta Concepción Núñez (en Burgos, 1989) aborda temas domésticos y de higiene, pero más tarde tratará cuestiones relacionadas con la defensa de la mujer y el reconocimiento de sus derechos.

Tras estas primeras experiencias, Carmen de Burgos continuará con sus colaboraciones en otros medios, tanto nacionales como internacionales: "la colaboración de Carmen de Burgos con la prensa será absolutamente asidua. El Heraldo de Madrid - para el que actúa como corresponsal de guerra en el conflicto marroquí a lo largo de 1909-, La Esfera, Nuevo Mundo, El Liberal, Por esos mundos, etc..." (Establier, 1997).

En 1905 Carmen de Burgos comienza a realizar su columna Femeninas en Heraldo de Madrid, que sustituye a la que realizaba para Diario Universal, y en la que continuará abordando temas relacionados con la situación de la mujer; y en 1909 llegó incluso a fundar su propio medio, Revista Crítica. La propia autora habla así de su trayectoria periodística en Al balcón:

"Mi labor de periodista es extensa, apasionada por todas las causas nobles. En la actualidad soy redactora del Heraldo de Madrid, colaboro en un centenar de periódicos, y dirijo Revista Crítica, fundada por mí que es la primera en tener una sección dedicada a los judíos. En sus columnas escriben con amor aquellos descendientes de los infelices que sufrieron la bárbara persecución que les arrojó de España en pasados siglos" (de Burgos, 1913:11).

\section{Metodología}

El trabajo que aquí se presenta plantea un análisis de las crónicas enviadas por Carmen de Burgos y publicadas en el Heraldo de Madrid durante los meses de agosto y septiembre de 1909, periodo en el cual la autora permaneció en las ciudades de Málaga, Almería y Melilla, dando cobertura informativa de la guerra de Marruecos. 
Para realizar el análisis se ha procedido a la revisión de todos los periódicos del Heraldo en ese periodo de tiempo y se han extraído los textos firmados por Colombine. Concretamente la muestra objeto de estudio es de un total de 15 crónicas.

La justificación de este objeto de estudio responde a dos motivos principalmente. El primero de ellos está relacionado con el ámbito periodístico, ya que dichas crónicas son el fruto del trabajo que desarrolló Carmen de Burgos durante unos meses y es precisamente este trabajo periodístico el que la hará pasar a la historia como la primera mujer española corresponsal de guerra. Y, por otra parte, Carmen de Burgos es considerada también como una de las primeras mujeres que fomentó el debate y la crítica en temas controvertidos que afectaron a la mujer de la época.

\subsection{Hipótesis y objetivos de investigación}

Dos son las hipótesis sobre las que asienta este estudio. La primera está relacionada con el ámbito periodístico propiamente dicho pues, partiendo de la idea de que Carmen de Burgos es considerada la primera mujer en ejercer profesionalmente como periodista y, por lo tanto, que ésta es una profesión desempeñada por hombres en su época, se contempla que las crónicas firmadas por Colombine ocupan dentro del periódico el Heraldo de Madrid información secundaria o poco relevante en relación a las crónicas firmadas por hombres.

Por otra parte, partiendo de la idea de que los distintos análisis realizados hasta el momento sobre la biografía y obra de Carmen de Burgos coinciden en considerarla como una de las primeras defensoras del papel social y cultural de la mujer, este trabajo plantea como segunda hipótesis que las citadas crónicas que la periodista realizó como corresponsal para cubrir la Guerra de Marruecos (1909) sirven de plataforma para visibilizar la situación de la mujer de la época y para reivindicar avances ideológicos en relación al concepto de derechos e igualdad de las mujeres.

Partiendo de estas hipótesis los objetivos generales de esta de investigación se centran en:

1. Analizar el tratamiento informativo que tienen las crónicas sobre la guerra de Marruecos, firmadas por Colombine dentro del Heraldo de Madrid (1909).

2. Analizar el tratamiento que hace Carmen de Burgos sobre la mujer en las crónicas de guerra que la periodista publicó en Heraldo de Madrid (1909).

A su vez estos objetivos generales se materializan en los siguientes objetivos específicos:

1. Determinar la importancia que tienen las crónicas de Colombine respecto al resto de textos publicados en el periódico, en cuanto a posición, tamaño y titular.

2. Determinar de qué manera se refiere la autora en sus textos a la figura femenina. 
3. Identificar si existen elementos críticos respecto al papel familiar, laboral y social de la mujer en aquella época.

La perspectiva metodológica a la hora de llevar a cabo el análisis planteado contempla en primer lugar un análisis cualitativo desde el punto de vista del tratamiento periodístico de las crónicas de Carmen de Burgos en su etapa como corresponsal de guerra, centrando la atención en aspectos como el emplazamiento de las informaciones, sus titulares y la presentación de las mismas; y en segundo lugar un análisis de contenido. Tal y como sostiene Berelson (1952) el empleo de un análisis del contenido de la información implica una descripción objetiva, sistemática y cuantitativa en el estudio del contenido de la comunicación, en este caso relacionado con el tratamiento de la mujer en dichas informaciones periodísticas.

\subsection{Planteamiento del análisis}

Para llevar a cabo el citado análisis se han tenido en cuenta una serie de variables que han sido aplicadas a las 15 crónicas objeto de estudio:

- En primer lugar, se ha procedido a la identificación de la información, para lo que se han contemplado aspectos como el periódico en el que se ha publicado la crónica (en este caso el Heraldo de Madrid exclusivamente), la fecha, la página y la extensión que ocupa dentro de dicha página (en número de líneas); además del antetítulo, título, subtítulo y cuerpo de la noticia.

- En segundo lugar, el tema que aborda la crónica, así como las acciones y los actores que la protagonizan.

- En tercer lugar, el papel que representa la mujer en cada una de las crónicas. En relación a este punto se han diferenciado seis roles (Herrero, 2010), que pueden encontrarse de forma individual o combinada:

1. Familiar: se incluyen en este grupo menciones al cuidado de los hijos, el papel como esposa, las relaciones de amistad y la realización de las tareas y labores en el hogar.

2. Personal y afectivo: aspectos como la belleza, el cuidado del cuerpo o su carácter y personalidad (si es cariñosa, alegre, cálida, sentimental, etc.).

3. Social: se consideran aquí todos aquellos aspectos relacionados con el entretenimiento, las compras, la moda, la vida pública, los actos sociales, etc.

4. Laboral: referido al trabajo que desempeñan las mujeres, la situación laboral y los salarios.

5. Educativo: alusiones a la instrucción y preparación de la mujer, la formación profesional y el nivel de estudios alcanzado.

6. Reivindicación para mejorar la situación de la mujer: se tienen en cuenta en esta variable las menciones a la mejora de la posición social de la mujer. 


\section{Análisis y resultados}

A principios del mes de agosto de 1909, Carmen abandonó la capital madrileña junto a su hermana Catalina para dirigirse a la costa andaluza y seguir de cerca el conflicto bélico realizando sus crónicas para el Heraldo de Madrid. Primero se establecerá en Málaga, desde donde envía su primera crónica, y posteriormente viajará a Almería, donde visitará hospitales informando sobre la actividad de la Cruz Roja y especialmente de las mujeres que ofrecían el servicio. En estas primeras crónicas (tabla I), Carmen aborda la situación de las dos ciudades andaluzas y de las iniciativas humanitarias que la población llevaba a cabo.

Cabe señalar que antes de su viaje a Andalucía, Carmen había realizado otros artículos en los que habla de las mujeres que colaboran con la Cruz Roja, concretamente se puede señalar una columna publicada el 1 de agosto de 1909 (en la cuarta página del Heraldo de Madrid de ese día) titulada Femeninas en la que la autora alaba la asistencia de la sección femenina de esta organización que ofrecen sus servicios en diferentes partes del mundo.

A finales de agosto de 1909, Colombine se traslada a Melilla, al mismo epicentro del conflicto, convirtiéndose de este modo en la primera mujer corresponsal de guerra en España. En Melilla continuará con su labor periodística dando noticia de todo lo que acontecía en el frente. Como señala Concepción Núñez, "realizó allí una labor humanitaria dando noticia de la situación de los soldados a los familiares que se lo solicitaban; también penetró en el mundo de la cultura mora, de la que hizo una descripción crítica, y en el ambiente del estamento militar español" (1992: 46). El Heraldo de Madrid tenía ya en Melilla a algunos de sus redactores como José Rocamora (que en 1909 será nombrado director del periódico), Luis Bonafoux o Luis de Armiñán que, desde el inicio del conflicto, daban cuenta de los detalles de las operaciones militares llevadas a cabo (Marín, 2013:14); pero la llegada de Carmen de Burgos supuso, por sí misma, un hecho noticioso que fue anunciado por El Telegrama del Rif, el 24 de agosto de 1909:

“En el vapor «Cabo Nao» llegó ayer la bella y notable escritora Carmen de Burgos, «Colombine», redactora de «Heraldo de Madrid», del cual ha recibido el importante encargo de estar al lado de la Cruz Roja de Melilla, dar cuenta de sus trabajos e informar a los lectores de aquel diario de cuanto a heridos o enfermos se refiera, proporcionando así datos al «Heraldo» para contestar a las peticiones de noticias que recibe. «Colombine» trae, pues, a Melilla, una hermosa misión que cumplir, y seguramente la llevará a cabo a la perfección, pues no otra cosa puede esperarse de su talento y su actividad. Sea bienvenida la distinguida periodista al teatro de la guerra".

Colombine envió siete crónicas al Heraldo de Madrid durante su estancia en Melilla (tabla I), la primera de ellas fechada el 24 de agosto -publicada el día 25- sobre los preparativos para la imposición de las cruces al Mérito militar a los oficiales y soldados heridos en el campo de batalla. No obstante, será el 30 de agosto cuando el Heraldo publique en portada la llegada de Carmen al continente africano, con el titular "«Colombine» en Melilla", con una crónica en la que ya la corresponsal informa 
de lo que sucede en los campamentos militares y de los últimos acontecimientos en el frente. Cabe destacar que el tratamiento que realiza esta periodista de la información está inevitablemente marcado por la importancia que adquirió "la cuestión de Marruecos" en la época (Marín, 2013). Tal y como afirma esta autora, la guerra fue concebida como un paso necesario e inevitable hacia el colonialismo y la explotación territorial, algo que queda reflejado en las crónicas enviadas por Carmen de Burgos, que están marcadas por un desenmascarado apoyo a la contienda y por una clara exaltación de la patria.

El trabajo de Carmen de Burgos como corresponsal de guerra se prolongó los meses de agosto y septiembre. Su última crónica sobre el conflicto la publicó el 19 de septiembre de 1909. Esta experiencia en el frente marcará su vida periodística. A su regreso a Madrid, todo el material que Carmen había elaborado sobre la guerra de Marruecos lo utilizó para la elaboración de un relato documental que tituló En la guerra (Episodios de Melilla), y que publicó el 29 de octubre en El Cuento Semanal (Núñez, 1992: 47).

\subsection{Análisis del tratamiento periodístico}

Las crónicas que Carmen de Burgos realizó en su periodo como corresponsal suelen comenzar con un antetítulo en el que se indica el lugar desde el que escribe la autora ("Desde Málaga", "Desde Almería" o "Desde Melilla"), a excepción de cuatro de ellas correspondientes a los días 12, 25 y 30 de agosto, y 27 de septiembre; no obstante en los dos primeros casos el lugar aparece citado inmediatamente después del titular, junto con la fecha del envío de la crónica al periódico, o en el caso del 30 de agosto aparece citado en el propio titular ("Colombine, en Melilla"). No sucede así con la última de las crónicas, del 27 de septiembre, donde es necesario leer el cuerpo de la noticia para saber desde dónde escribe la autora. En las otras 11 crónicas en las que sí que aparece mencionado el lugar éste viene indicado en mayúsculas, a modo casi de titular, ya que en algunas de ellas tiene un cuerpo de letra mucho mayor al del propio titular. Esto no es algo exclusivo de las informaciones publicadas por Carmen de Burgos, sino que forma parte del diseño del propio Heraldo de Madrid donde es frecuente que las crónicas aparezcan tituladas por el lugar desde el que se envían ("En el Peñon", 14 de agosto de 1909; "Desde Ceuta", 18 de agosto de 1909; "Desde Tetuán”, 27 de septiembre de 1909).

Cabe señalar, por otra parte, en relación a los titulares de las crónicas de Colombine que no existe como tal un titular periodístico propiamente dicho, sino que está compuesto por frases cortas, separadas por guiones, que a modo de telegrama resumen el contenido de la información ("Hablando con la marquesa de Polavieja, -Por los hospitales. -La asistencia de heridos.", 10 de agosto de 1909). No obstante, se pueden señalar dos excepciones donde sí que podría hablarse de la existencia de un titular: es el caso de las crónicas del 9 de agosto ("Servicios de la Cruz Roja") y del 27 de septiembre ("Una mora del harén del Roghi"). 
Es importante mencionar, además, que todas las crónicas aparecen firmadas por Colombine, en letra negrita al final de la información, y en cinco de ellas (de los días $9,10,11,18$ y 20 de agosto) aparece la frase "De nuestra redactora «Colombine»" al principio del texto, ya sea antes o después del titular. Más importante que el número de veces en las que aparece esta referencia de Colombine como "redactora" es el hecho de que el propio medio de comunicación la otorga este estatus dentro de la redacción, algo que la sitúa al mismo nivel que el resto de los redactores de la plantilla del Heraldo de Madrid.

Por otro lado, de las 15 crónicas de guerra publicadas por Carmen de Burgos, cuatro fueron enviadas por la periodista desde Málaga durante las primeras semanas de su trabajo, cuatro desde Almería cuando se trasladó a esta ciudad con su hermana $\mathrm{y}$, finalmente, seis crónicas pertenecen al periodo en el que actuó como corresponsal desde Melilla, tal y como puede apreciarse en la tabla I. Esta creciente proximidad de Carmen de Burgos respecto a la guerra influye decisivamente en la colocación de sus crónicas dentro de las páginas del periódico. En el caso de los artículos escritos desde Málaga, se observa que las informaciones que envía Carmen ocupan un lugar secundario con respecto a las informaciones de sus compañeros de redacción, quedando relegadas a la segunda, tercera o incluso cuarta página (a excepción de una de ellas, publicada el 20 de agosto, que aparece en portada). Pero a partir de su traslado a Almería, y especialmente tras su viaje a Melilla, las crónicas de Colombine adquieren un mayor protagonismo dentro del Heraldo, de modo que en la mayoría de ocasiones aparecen situadas en la primera página del periódico (exceptuando dos que se sitúan en la segunda y tercera página). En este sentido, resulta significativo comprobar cómo el número de líneas que ocupan las crónicas de la periodista en el Heraldo de Madrid asciende a medida que Carmen se acerca al lugar de la batalla. En la tabla I se puede comprobar que durante su estancia en Málaga y gran parte del tiempo que pasó en Almería, los textos publicados ocupan menos de 100 líneas. Sin embargo, a partir de su llegada a Melilla, la posición privilegiada de sus crónicas se ve acompañada por un considerable aumento de su extensión (entre 150 y 227 líneas).

Este cambio en el emplazamiento de los textos elaborados por Carmen de Burgos y la creciente extensión de sus crónicas estarían relacionados por tanto con la proximidad de la redactora al epicentro del conflicto, que hace que su trabajo adquiera más interés para el medio, más que por la autoría de las mismas. 
Tabla 1. Resumen del análisis del tratamiento periodístico en las crónicas de Carmen de Burgos durante la Guerra de Marruecos. Fuente. Elaboración propia.

\begin{tabular}{|c|c|c|c|c|c|}
\hline $\begin{array}{c}\text { Fecha } \\
\text { Colombine }\end{array}$ & $\begin{array}{l}\text { Fecha } \\
\text { publicación } \\
\text { Heraldo de } \\
\text { Madrid }\end{array}$ & $\begin{array}{l}\text { Lugar desde el } \\
\text { que se escribe la } \\
\text { crónica }\end{array}$ & Página & $\begin{array}{l}\text { Tamaño de } \\
\text { la crónica } \\
\text { ( } \mathrm{n}^{\circ} \text { líneas) }\end{array}$ & Crónica \\
\hline 8 de agosto & 9 de agosto & Málaga & Pág. 2 & 64 & Desde Málaga. Servicios de la Cruz Roja. \\
\hline 9 de agosto & 10 de agosto & Málaga & Pág. 2 & 112 & $\begin{array}{l}\text { Desde Málaga. Hablando con la marquesa de } \\
\text { Polavieja. -Por los hospitales. -La asistencia } \\
\text { de heridos. }\end{array}$ \\
\hline 10 de agosto & 11 de agosto & Málaga & Pág. 3 & 66 & $\begin{array}{l}\text { Desde Málaga. Preparativos de la Cruz Roja. - } \\
\text { Visitando a los heridos. -Donativos. - } \\
\text { Peticiones justas. }\end{array}$ \\
\hline 11 de agosto & 12 de agosto & Málaga & Pág. 4 & 56 & $\begin{array}{l}\text { Notas de "Colombine". Los barracones de la } \\
\text { Trinidad. -Preparativos de la Cruz Roja. - } \\
\text { Heridos a Melilla. }\end{array}$ \\
\hline- & 14 de agosto & Almería & Pág. 2 & 53 & $\begin{array}{l}\text { Desde Almeria. Preparativos de Málaga. - } \\
\text { Tristeza en Almeria. - Sin fiestas. -El hospital } \\
\text { de sangre. -En previsión de una epidemia. }\end{array}$ \\
\hline - & 18 de agosto & Almería & Pág. 4 & 74 & $\begin{array}{l}\text { Desde Almeria. Entusiasmo patriótico. - } \\
\text { Ofrecimientos. -El servicio de correos y } \\
\text { telégrafos. -Cartas del público. }\end{array}$ \\
\hline- & 20 de agosto & Almeria & Portada & 57 & $\begin{array}{l}\text { Desde Almeria. Preparando un hospital de } \\
\text { sangre. -Una «kermesse». -Iniciativas } \\
\text { humanitarias. }\end{array}$ \\
\hline- & 21 de agosto & Almería & Pág. 4 & 39 & Desde Almeria. Las damas de la Cruz Roja. \\
\hline 24 de agosto & 25 de agosto & Melilla & Pág. 2 & 32 & $\begin{array}{l}\text { Telegrama de "Colombine". La Cruz Roja en } \\
\text { Melilla. -Preparativos. -Imposición de cruces. }\end{array}$ \\
\hline 26 de agosto & 30 de agosto & Melilla & Portada & 167 & $\begin{array}{l}\text { Por los campamentos. "Colombine" en } \\
\text { Melilla. Recorriendo las tiendas. -Los } \\
\text { voluntarios. -Ingleses que aman a España. - } \\
\text { Fiesta en el campamento. -Interrumpe el } \\
\text { tiroteo. -A las avanzadas. -silbando las balas. } \\
\text {-Torna la calma. -A la plaza. }\end{array}$ \\
\hline 31 de agosto & 2 de septiembre & Melilla & $\begin{array}{l}\text { Portada } \\
\text { y pág. } 2\end{array}$ & 221 & Desde Melilla. En el Dchar. \\
\hline - & 9 de septiembre & Melilla & Pág. 3 & 150 & $\begin{array}{l}\text { Desde Melilla. Visitando hospitales. Hace falta } \\
\text { un buen local. - Los epitafios diseminados. -En } \\
\text { el central. -Escena dolorosa. -Elogios al } \\
\text { personal. -El corneta sordomudo. -Dos moros } \\
\text { heridos. -Hablando con uno de ellos. -El } \\
\text { número de enfermos y heridos actualmente. }\end{array}$ \\
\hline- & 10 de septiembre & Melilla & Portada & 227 & $\begin{array}{l}\text { Desde Melilla. El domingo en el campamento. } \\
\text {-Misa y cañonazos. -Diversiones dominicales. } \\
\text {-Una orquesta árabe. }\end{array}$ \\
\hline - & 19 de septiembre & Melilla & Portada & 157 & $\begin{array}{l}\text { Desde Melilla. El té de las cinco. -Una mesa } \\
\text { original. -El té árabe. -Costumbres moras. } \\
\text { Sentimentalismo mal empleado. }\end{array}$ \\
\hline - & 27 de septiembre & - & Portada & 108 & Una mora del harén del Roghi. \\
\hline
\end{tabular}




\subsection{Tratamiento de la mujer en las crónicas de Colombine}

En las crónicas analizadas los protagonistas de las informaciones son tanto hombres como mujeres. Destaca que el caso de los hombres éstos aparecen con nombres y apellidos, además de sus profesiones. Se ha encontrado con frecuencia menciones a Antonio Gómez Díaz (presidente de la Cruz Roja), el doctor Calatraveño, el General Marina, escritores (como Narciso Díez Escobar y Ramón A. Urbano) y periodistas (como Enrique Rivas, Luis Salado y Leopoldo Romeo).

En el caso de las mujeres encontramos pocos nombres propios. Se menciona, por ejemplo, a la marquesa de Polavieja (Concepción Benjumea de Polavieja), una mujer que desempeñaba un papel activo en la vida social de la época, fundamentalmente relacionado con causas benéficas. También se menciona a la marquesa de Squilache, María del Pilar de León y Gregorio, que había sido embajadora consorte de España en las legaciones diplomáticas de Washington y Constantinopla y era formaba parte de la Junta de Damas de la Cruz Roja (Gay Heredia, 2012). Por último, Carmen también se refiere a la ex Emperatriz Eugenia de Montijo, que además de haber desarrollado un papel político activo durante el mandato de su esposo, Napoleón III, era Dama honoraria Gran Cruz de la Orden del Imperio Británico.

No obstante, sí que encontramos muchas referencias a las mujeres, en especial a aquellas que prestan sus servicios en la Cruz Roja, a las que Carmen se refiere como: damas de la Cruz Roja (o simplemente "damas"), Junta de señoras, Comisión de señoras, sección de damas o señoras de la Cruz Roja. Pero también aparecen otras menciones a las mujeres a las que se refiere genéricamente como señoras, madres, hermanas, esposas, mujeres españolas, damas francesas, la mujer, señoras malagueñas.

En sus textos, Carmen aborda a menudo temas relacionados con las mujeres en los que éstas son las protagonistas o forman parte, de alguna otra manera, en el relato. Concretamente, en las 15 crónicas analizadas hemos encontrado los siguientes temas relacionados con la figura de la mujer:

- Celebración de la Junta de Damas de la Cruz Roja presidida por la marquesa de Polavieja.

- Entrevista a la marquesa de Polavieja.

- Descripción de la misión de las señoras de la Cruz Roja.

- Correspondencia de mujeres españolas que querían colaborar con la Cruz Roja.

- Más de 30 mujeres visitan a los heridos en el hospital.

- Donaciones realizadas por mujeres a la Cruz Roja.

- Preocupación de las madres por sus hijos en la guerra.

- Actos de recaudación para la guerra organizados por las mujeres de clase alta.

- Constitución de la Junta Directiva de las Damas de la Cruz Roja. 
- Recuerdos de los soldados a la figura femenina (madres, hermanas, amantes).

- Analogía de la guerra con la figura de la mujer.

- Encuentro con mujeres moras en un Dchar: descripción física y del carácter de las mujeres marroquíes.

- La mujer como elemento esencial en los procesos políticos y sociales.

- Descripción de la dura vida de las mujeres marroquíes.

- Envío de cartas románticas de mujeres españolas a hombres marroquíes.

- Esclavitud y figura femenina en Marruecos.

- Descripción física y del carácter de una mora que formó parte de un harén.

En cuanto a la aparición de los seis roles con los que puede asociarse la mujer (Herrero, 2010) en las crónicas de Carmen de Burgos se puede señalar lo siguiente:

a) Rol Familiar

\section{Rol familiar}

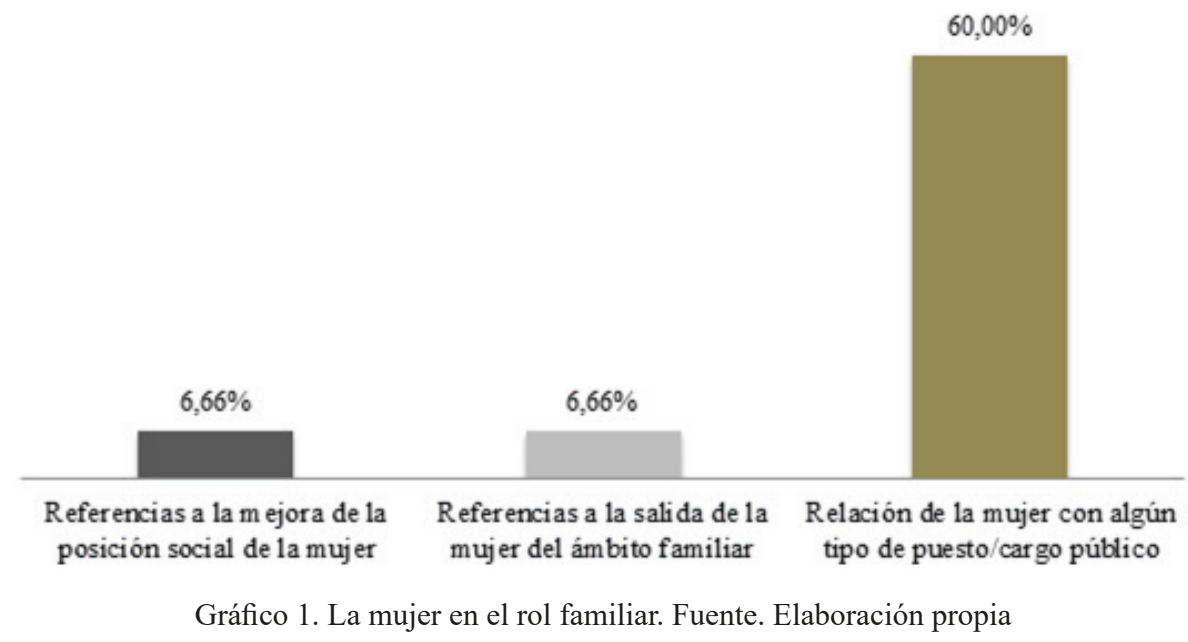

Carmen de Burgos se refiere a la mujer como esposa en 7 de las crónicas y en un número menos significativos de ocasiones la asocia al cuidado de los hijos (3), a la realización de tareas del hogar (3) y a las relaciones de amistad (3). Es importante señalar que, aunque no se cita explícitamente cuando Carmen habla del cuidado de los heridos por parte de las mujeres de la Cruz Roja hace alusión al punto de vista maternal. 
b) Rol personal y afectivo

La figura femenina aparece asociada a la imagen física y cuidado del cuerpo en 6 crónicas ( $40 \%$ de las crónicas totales), como es el caso por ejemplo de la publicada el 2 de septiembre: “¡Oh espíritu femenino! ¡Siempre el mismo! Han sacrificado sus rizos de azabache por el deseo de embellecerse más, y sufren el martirio de los enormes aretes y los tatuajes para parecer más lindas".

Por otro lado, el carácter asociado a las mujeres viene definido por adjetivos como tierna, cariñosa, elegante, distinguida, complaciente, caritativa, digna, alegre, entusiasta, curiosa, sensible, coqueta, sentimental, romántica, celosa e impaciente.

En este sentido, es necesario destacar que durante su estancia en Melilla Carmen de Burgos tiene la oportunidad de relacionarse con mujeres marroquíes en diferentes ocasiones y las describe en algunas de sus crónicas con algunos adjetivos que coinciden con los señalados anteriormente (impacientes, tiernas, dignas, curiosas), pero en el caso de "las moras" también incluye otros calificativos como expresivas, amables, respetuosas, medrosas, recatadas, melancólicas o serias. Es importante destacar que, en su última crónica desde Melilla, Una mora del harén de Roghi, narra la historia de una mujer que tras la huida de 'su señor' trabaja como sirviente, y la describe con palabras como fría, fresca, vulgar, primitiva o semisalvaje: "Aquella mujer primitiva, semisalvaje era un documento más interesante para el psicólogo que uno de nuestros pequeñuelos niños europeos".

c) Rol social

\section{Rol social}

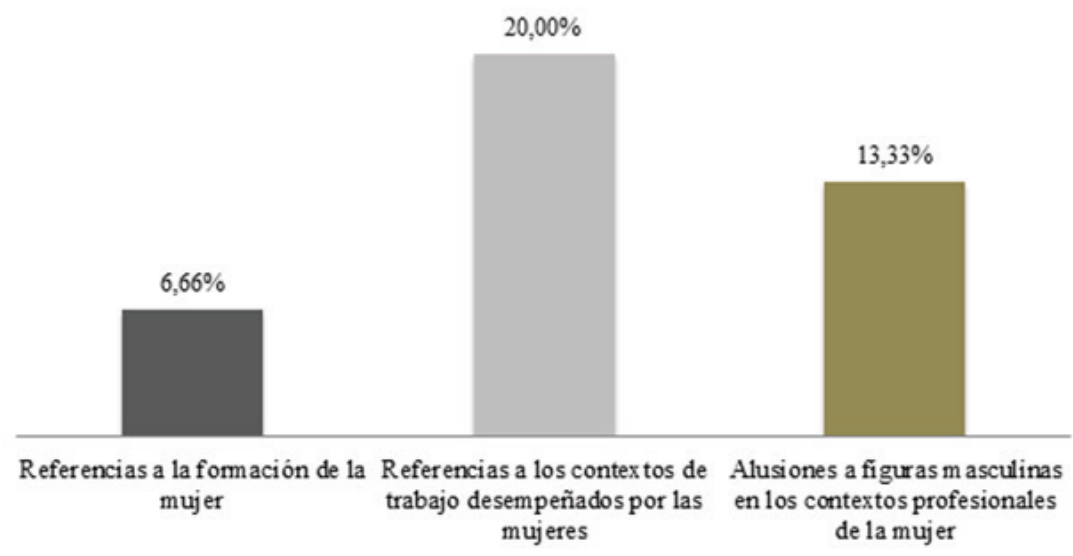

Gráfico 2. La mujer en el rol social. Fuente. Elaboración propia 
En cuanto al papel social desempeñado por la mujer, en 6 de las crónicas aparecen en actos sociales (como tomar el té o leer la prensa), pero sólo en 3 ocasiones acude la mujer sola a este tipo de eventos, siendo lo habitual que aparezca junto con otras personas (que pueden ser hombres o mujeres ya que las visitas a los hospitales que realizan las "damas" de la Cruz Roja se realizan en grupo).

d) Rol laboral

\section{Rol laboral}

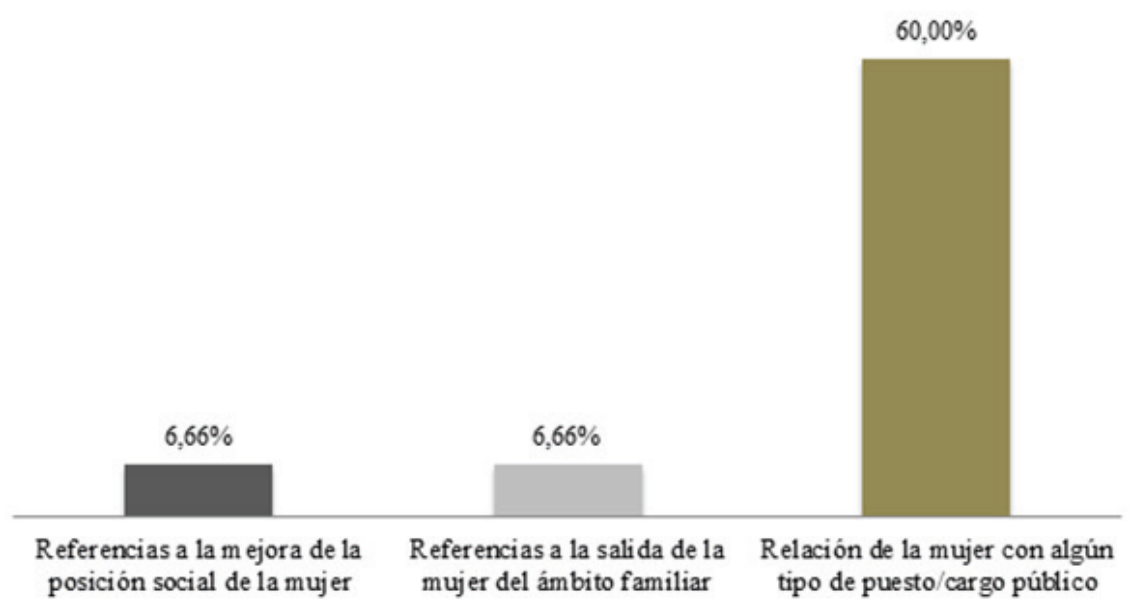

Gráfico 3. La mujer en el rol laboral. Fuente. Elaboración propia

Por otro lado, Carmen de Burgos hace algunas menciones al ambiente profesional de la mujer, siendo los trabajos que se asocian a la figura femenina en estas crónicas los de periodista, enfermera y sirvienta. El trabajo de enfermera en este caso se vincula con el cuidado de los heridos, algo que resume la periodista en su publicación el 10 de agosto: "hablando de la alta misión de la mujer al lado de los heridos, que no se reduce solo al auxilio material, sino al consuelo que es para ellos y para sus familias esa prolongación del hogar que el alma femenina lleva siempre consigo (...)". Pero además se asocia con las mujeres otra tarea que es la de ser el enlace entre los soldados y sus familias: "Las señoras de la Cruz Roja, de acuerdo con los señores de la Comisión provincial, se encargan de escribirles cartas a los soldados, de ponerles telegramas a las familias, de facilitarles sin que cueste nada todos los medios de comunicación" (crónica del 10 de agosto de 1909).

e) Rol educativo

Respecto a la educación de la mujer, no se han encontrado referencias significativas a su formación ni a su nivel de estudios, a excepción de la crónica del día 18 de agosto, en la que se alude a las alumnas de la Escuela de Artes e Industrias. 


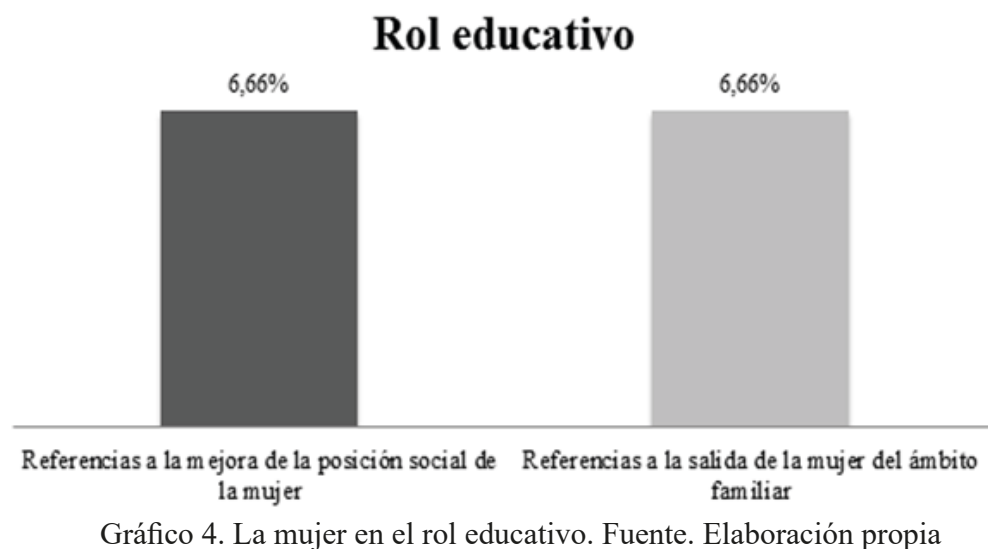

f) Reivindicaciones para mejorar la posición de la mujer

Por último, en cuanto a la reivindicación de una mejor posición (social y laboral) para la mujer, se ha observado que en 9 de las crónicas se relaciona la mujer con algún tipo de puesto público (por ejemplo, los cargos dentro de la Junta Directiva de las Damas de la Cruz Roja, la marquesa de Squilache o la ex Emperatriz Eugenia). Es interesante destacar que en 5 crónicas se menciona a la marquesa de Polavieja, pero nunca aparece el nombre de dicha marquesa.

\section{Reivindicaciones derechos e igualdad de la mujer}

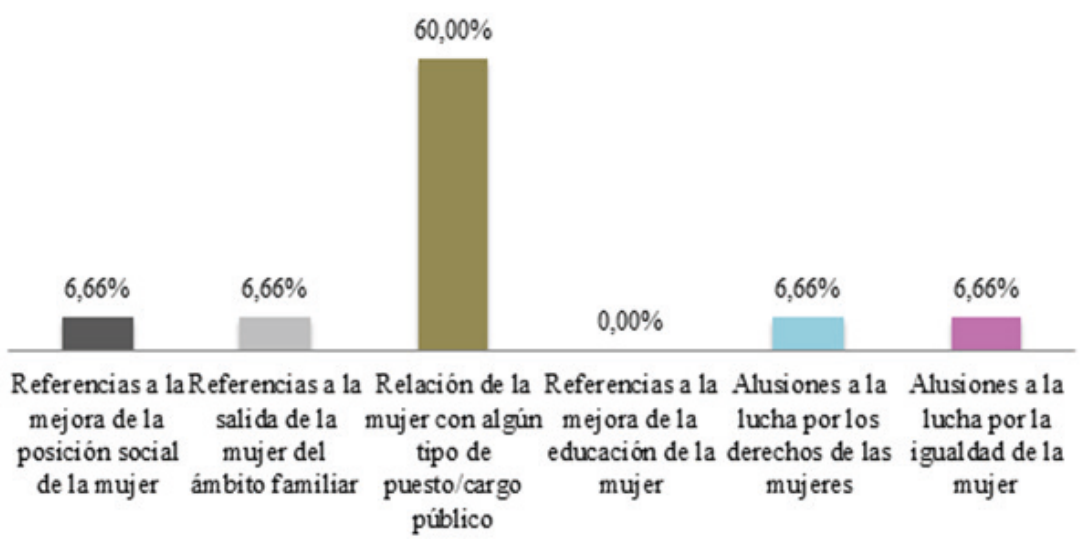

Gráfico 5. Reivindicaciones para la mejora de la posición de la mujer. Fuente. Elaboración propia 
Asimismo, también existen en las crónicas de Colombine referencias significativas a la mejora de la posición de la mujer y a su lucha por la igualdad, como el último párrafo del texto publicado el día 2 de septiembre de 1909: “... pienso qué elemento tan poderoso podía ser la mujer tomando parte, como lo han hecho las francesas, en la obra de la civilización de los pueblos...”. También aparece una reivindicación del papel de las mujeres como madres en la crónica del 18 de agosto ("madres a la cabecera del que sufre"), y también otro tipo de reivindicaciones dirigidas a poner en valor las cualidades de la mujer y a dignificarlas, como es el caso de la crónica del 30 de agosto que equipara la guerra con la figura de la mujer. Si bien estas referencias no son abundantes, es preciso destacar su importancia dado el contexto histórico en el que se publicaron.

\section{Conclusiones}

Tras abordar el análisis de las crónicas de guerra de Carmen de Burgos, la primera hipótesis sobre la que se asienta el presente estudio queda refutada. Es decir, a pesar de que Carmen de Burgos es la primera periodista y reportera de guerra, el Heraldo de Madrid le ofrece desde el principio el mismo tratamiento que a cualquier otro de sus redactores, y resulta especialmente significativa la prioridad que reciben sus crónicas sobre la Guerra de Marruecos a nivel de portada y extensión.

A pesar de que el género periodístico de la crónica tiene como objetivo describir la situación que atraviesan los soldados españoles en la guerra, en sus textos Carmen de Burgos aprovecha para visibilizar la situación de la mujer, tanto en España como en Marruecos. En este sentido, resulta cuando menos chocante el modo en que describe a "las moras" cuando se refiere a ellas como mujeres "primitivas" y "semisalvajes", pero hay que destacar que al mismo tiempo en sus textos aborda la posición de sumisión de las mujeres marroquíes respecto al hombre y pone así énfasis en una realidad hasta entonces casi ignorada. De esta manera, tras el análisis es posible corroborar que Carmen de Burgos no sólo desarrolló su labor como periodista durante la Guerra de Marruecos, sino que, además de informar, sus crónicas sirvieron de plataforma para reivindicar una mejora de los derechos e igualdad de las mujeres, aspectos que constatan la segunda hipótesis en la que se basa este estudio.

\section{Referencias bibliográficas}

BERELSON, B. (1952). Content Analysis in Communication Researches. Glencoe III, Free Press.

BERNÁRDEZ, A.; ANDRÉS, J. DE; LORENZO, J. Y VARGAS, A. (2007). Escritoras y periodistas en Madrid (1876-1926). Área de Gobierno de Empleo y Servi- 
cios a la Ciudadanía. Dirección General de Igualdad de Oportunidades. Ayuntamiento de Madrid.

BURGOS, C. DE. (1989). La flor de la playa y otras novelas cortas. Madrid: Editorial Castalia.

BURGOS, C. DE. (1904). El divorcio en España. Madrid: Viuda de Rodríguez Serra. (1913). Al balcón. Valencia: Sempere.

CABANILlAS, Á. (2006). Carmen de Burgos "Colombine", crítica feminista de arte. Espacio, Tiempo y Forma, 18-19. Doi: 10.5944/etfvii.18-19.2005.1490

DALE, S. (2005). La Pensadora gaditana por Doña Beatriz Cienfuegos. Newark: Juan de la Cuesta.

El Telegrama del Rif (1909). Carmen de Burgos (noticia publicada el 24 de agosto). Recuperado de http://goo.gl/uCSNQj el 19 de abril de 2016.

ESTABLIER, H. (1997). Mujer y feminismo en la narrativa de Carmen de Burgos (tesis doctoral, Universidad de Alicante). Recuperado de http://goo.gl/Jf2abV el 9 de abril de 2016.

GAY HEREDIA, A. (2012). La Marquesa de Squilache (Benefactora de la ciudad de Motril). Recuperado de http://decastroero.blogspot.com.es/2012/05/la-marquesa-de-squilache-benefactora-de.html el 21 de junio de 2016.

HERRERO, R. (2010). La imagen de la mujer en la prensa entre 1910-1915 y 20002005: estudio comparado (tesis doctoral, Universidad Complutense de Madrid). Recuperado de http://goo.gl/ezcNjr el 21 de abril de 2016.

LATORRE, N. (1998). Carmen de Burgos "Colombine". Asparkía, IX. Recuperado de http://goo.gl/k2c7wO el 9 de abril de 2016.

MARÍN, M. (2013). Colonialismo, género y periodismo. Cuatro mujeres españolas en las guerras con Marruecos (1909-1927): Carmen de Burgos, Consuelo González Ramos, Teresa Escoriaza y Margarita Ruiz Lihory. Revista Clepsydra, 12. Recuperado de https://goo.gl/qV7lkx el 11 de abril de 2016.

MINESSO, B. (2011). Malcasadas vencidas y liberadas. Anhelos de cambio en la narrativa de Carmen de Burgos, "Colombine". Cuadernos de Aleph, 3. Recuperado de https://goo.gl/rqRpHo el 27 de abril de 2016.

NúÑEZ, C. (1992). Carmen de Burgos, Colombine (1867-1932). Biografia y obra literaria (tesis doctoral, Universidad Complutense de Madrid). Madrid: Editorial de la Universidad Complutense de Madrid.

(2012). La escritora y periodista Carmen de Burgos, corresponsal en la guerra de España y Marruecos (1909). Candil: Revista del Hispanismo-Egipto, 12. Recuperado de https://goo.gl/WUDUxx el 9 de abril de 2016.

PEDRO, C. DE (2014). Ángeles de la guerra. Los discursos pacifistas de Carmen de Burgos y Sofía Casanova tras sus experiencias como corresponsales. En E. González \& M. González (Eds.) Mujeres en guerra/guerra de mujeres en la sociedad, el arte y la literatura. Sevilla: Arcibel editores. Recuperado de https://goo.gl/ PhY9cH el 11 de abril de 2016.

RODRÍGUEZ, N. (2007). La imagen de la mujer en la publicidad gráfica en España en el primer tercio del siglo XX. In Quintas Jornadas Imagen, Cultura y Tecnolo- 
gía:[celebradas durante los días 3, 4 y 5 de julio de 2006] (pp. 383-400). Editorial Archiviana.

SANZ, A. (2010). Hasta que la ley o el divorcio nos separe. ARBOR Ciencia, Pensamiento y Cultura, 186 (extra). doi: 10.3989/arbor.2010.extrajunion3007

SCANLON, G. (1986). La polémica feminista en la España contemporánea, 18681974 (Vol. 4). Madrid: Akal.

SEVILlANO, A. y SEGURA, A. (2009). Carmen de Burgos "Colombine" (Almería, 1867-Madrid, 1932). Instituto de Estudios Almerienses: Colección Historia, 26.

ZAPATA-CALLE, A. (2011). En la guerra de Carmen de Burgos: Crítica del proceso de nacionalización e imperialismo español en Marruecos. Decimonónica, 8(2). Recuperado de http://goo.gl/FYkxvN el 11 de abril de 2016. 